\title{
Workshop on Compressible Multiphase Flows Derivation, closure laws, thermodynamics
}

The first Workshop on Compressible Multiphase Flows was held in Strasbourg (France), in May 2018, 23rd-25th. The goal was to address the modelling of compressible multiphase flows, gathering specialists of this subject, who came from math departments of universities but also from the industry.

The participants shared modelling methods, difficulties, (rigorous or more phenomenological) analysis, allowing for the description of multiphase flows with exchanges (mass transfer, energy exchange...) and apparition of shock waves. The main topics were:

- The Physics of multiphase flows with mass transfer and high energy exchanges;

- The derivation and analysis of PDE models for compressible multiphase flows;

- The Construction of coherent thermodynamical laws.

Note that one-velocity and multi-velocity models were considered, while numerical aspects were disregarded in order to focus on fruitful discussions on the modelling issues.

The details of the workshop, such as the list of speakers and the title of their talks, can be found here:

http://gdr-manu.math.cnrs.fr/Thermo2018.php

The workshop received financial support from EDF R\&D, GdR MaNu, Université de Strasbourg.

As co-editors of these ESAIM Proceedings, we are grateful to all the authors who have contributed to this volume. We also thank all the referees for their valuable comments which helped improving the final version of the papers. The ESAIM editorial team is also warmly acknowledged.

Philippe Helluy, Jean-Marc Hérard, Nicolas Seguin. 